\title{
Project Title: Radiochemical Analysis by High Sensitivity Dual-Optic Micro X-ray Fluorescence
}

DOE Report Number: 81967

Publication Date:

Lead Principal Investigator:

George J. Havrilla

Los Alamos National Laboratory

LANL MS G740

P.O. Box 1663

Los Alamos, NM 87545-1663

505-667-9627

havrilla@lanl.gov

Co-Investigator: (repeatable)

Ning Gao

X-Ray Optical Systems

15 Tech Valley Drive

East Greenbush, NY 12061

518-880-1500

ngao@xos.com

Graduate Students - 0

Research Objective

A novel dual-optic micro X-ray fluorescence instrument will be developed to do radiochemical analysis of high-level radioactive wastes at DOE sites such as Savannah River Site and Hanford. This concept incorporates new X-ray optical elements such as monolithic polycapillaries, which focus X-rays. The polycapillary optic can be used to focus X-rays emitted by the X-ray tube thereby increasing the X-ray flux on the sample over 1000 times. The polycapillary optic will also be used to collect the $\mathrm{X}$-rays from the excitation site. This will effectively screen the radiation background from the radioactive species in the specimen. This dual-optic approach significantly reduces the background and increases the analyte signal thereby increasing the sensitivity of the analysis. This dual-capillary design is essentially a confocal (having the same foci) design, i.e. the detected X-rays are only emitted from the overlap of the two focal spots. This increases spatial resolution and reduces background. The integration of the X-ray optics increases the signal-to-noise and thereby increases the sensitivity of the analysis for low-level analytes. This work will address a key need for radiochemical analysis of high-level waste using a non-destructive, multi-element, and rapid method in a radiation environment. There is significant potential that this instrumentation could be capable of on-line analysis for process waste stream characterization at DOE sites.

\section{Research Progress and Implications}


As of May 2004 this report summarizes work from June 2003 through May of 2004, year 3 of a 3 -year project. The project is behind the original milestone timetable due to issues in constructing and getting the prototype instrument operational at LANL for testing.

Experiments performed in the summer of 2003 with the prototype core demonstrated the key features of the initial design: desired sensitivity and radiation rejection. The side benefit of a confocal X-ray microscope was also demonstrated.

The prototype core instrument was tested with the model analyte system ( $\mathrm{Sr}, \mathrm{Pt}, \mathrm{Pb}, \mathrm{Pd}, \mathrm{Mo}, \mathrm{Cu}$ ) utilizing $1 \mathrm{M}$ sodium chloride as a test matrix for the anticipated sodium hydroxide matrix from the sites. The model analyte system was a 100-ppm concentration solution of the elements listed above. All of the target elements were detected below 10-ppm detection limit with a 600-second counting time except for palladium. The Pd had a detection limit of 26 ppm due to excitation issues with the silver $\mathrm{X}$-ray tube. An iron 55 source was used to determine the radiation background reduction with the dual capillary (confocal) optic design. The radiation rejection measurements showed a factor of 20 rejection ratio using an iron 55 source.

The prototype core was incorporated into a chamber designed for a prototype instrument. The instrument is equipped with shielding, a window to view the instrument, interlocked doors, power supply and sample stage control all interfaced with a personal computer. The software control was developed by XOS. Data acquisition is accomplished via a Lab View interface. The instrument was delivered to LANL in early May 2004, a few months past the original schedule delivery of January 2004. The delay was due to design issues and parts availability.

One of the more significant developments of this project is the realization that this instrument is a confocal X-ray microscope. The overlap of the two focal regions of the excitation and detection optics creates a confocal volume which can be scanned in 3-dimensions. The significance of this achievement is the ability to acquire 3-D elemental maps, nondestructively. Prior to this achievement, the only way to acquire such elemental information was to use a synchrotron. Now such 3D elemental information can be obtained in a laboratory setting.

The confocal capabilities were demosntratred through two significant experiments: 1) depth profile through a 13 layer paint chip and 2) elemental mapping of iron particles in a boron nitride pellet. The paint chip demonstration shows the layers of paint through the entire paint chip, almost 1000 micrometers thick. Different elements appeared at different depths showing the changes in paint particularly the use of lead years ago. The elemental mapping of the iron particles showed both a 2D distribution of iron in the x,y plane of the scan, as well as a 3D distribution of iron to a depth of 750 micrometers.

Presentations on the confocal MXRF instrument were given at the Denver X-ray conference in Colorado Springs, CO, August 2003 and at the MRS Fall Meeting in Boston, MA, December 2003.

Due to the unique nature of this new capability an R\&D 100 Award package was prepared and submitted for competition in March 2004. On June 29, 2004, we were notified that the confocal X-ray microscope was selected as one of the R\&D 100 winners for 2004. 
An extension proposal was submitted to complete the limited hot testing to run from October 2004 through March 2005.

\section{Planned Activities}

07/01/04 - 09/30/04 - testing and evaluation of prototype at LANL

Tentative with extension funding:

10/01/04 - 03/31/05 - controlled hot testing at LANL

Information Access

OPTIONAL ADDITIONAL INFORMATION OPTIONAL PROPRIETARY INFORMATION 